\title{
Preoperative Administration of Excess Iodide Increases Thyroid Volume of Patients with Graves' Disease
}

\author{
TOMONori YABUTA, Yasuhiro ITO, Mitsuyoshi HIROKAWA*, Mitsuhiro FUKUSHIMA, \\ Hiroyuki INOUE, Chisato TOMODA, TAKUya HIGASHIYAMA, Minoru KIHARA, TAKashi URUNO, \\ YuUKi TAKAMURA, KaORU KOBAYASHI, AKIHIRO MIYA, Fumio MATSUZUKA AND AKIRA MIYAUCHI
}

Department of Surgery, Kuma Hospital, 8-2-35, Shimoyamate-dori, Chuo-ku, Kobe 650-0011, Japan

* Department of Pathology, Kuma Hospital, 8-2-35, Shimoyamate-dori, Chuo-ku, Kobe 650-0011, Japan

\begin{abstract}
Preoperative excess iodide administration for patients with Graves' disease has been widely adopted by surgeons to perform surgery safely, because it decreases blood flow in the thyroid. However, surgeons often encounter the enlargement of thyroid volume after iodide administration, which makes surgery even more difficult. In this study, we retrospectively investigated the change in thyroid volume in Graves' disease that was evaluated on ultrasonography between before and after iodide administration. Eighty-nine patients who received iodide administered $(\mathrm{KI}(+)$ patients) and 24 in whom iodide was not administrated (KI(-) patients) before surgery for Graves' disease were enrolled in the study. The level of free T4 (FT4) significantly decreased and that of thyroid stimulating hormone (TSH) significantly increased after iodide administration. Average thyroid volume also significantly increased for $\mathrm{KI}(+)$ patients after iodide administration and $17 \%$ of these patients showed a $30 \%$ or more increase in thyroid volume. In KI(-) patients who were preoperatively treated only by anti-thyroid drugs, thyroid volume did not change before surgery. Preoperative TSH levels remained below measurement sensitivity in 37 of $\mathrm{KI}(+)$ patients, but the average thyroid volume also significantly increased after iodide administration. These findings suggest that thyroid volume in Graves' disease can increase with iodide administration not only due to TSH stimulation but also due to reasons other than TSH. Surgeons should be careful when preoperatively iodide administering to patients with Graves' disease, especially when the goiter is large.
\end{abstract}

Key words: iodide administration, surgery, Graves' disease, thyroid volume

(Endocrine Journal 56: 371-375, 2009)

PREOPERATIVE excess iodide administration is a widely used strategy for surgery on Graves' disease. This strategy was first advocated in the 1920s [1,2] and has been widely adopted by surgeons in order to perform operations safely. To date, many studies have demonstrated that iodide inhibits blood flow in the thyroid gland in euthyroid humans and in patients with Graves' disease using various investigative approaches, including evaluation of intrathyroid blood flow on ultrasonography and of microvessel density [3-9]. The mechanisms underlying blood flow reduc-

Received: August 28, 2008

Accepted: December 26, 2008

Correspondence to: Yasuhiro ITO, M.D., Ph.D., Department of Surgery, Kuma Hospital, 8-2-35, Shimoyamate-dori, Chuo-ku, Kobe 650-0011, Japan tion by iodide are unknown. Recently, Yamada et al. showed that iodide inhibits the expression of some genes related to angiogenesis, such as vascular endothelial growth factor (VEGF)-A, VEGF-B, and placental growth factor (PGF) in cultured human thyroid follicles from patients with Graves' disease and speculated that the downregulation of these factors at least partly accounts for the iodide-induced decrease in thyroid blood flow [10]. In contrast, Namba et al. demonstrated that iodide administration increased the thyroid volume of 10 male euthyroid volunteers together with significant increases in serum thyroid stimulating hormone (TSH) level [11]. Furthermore, surgeons often encounter thyroid enlargement with Graves' disease patients after iodide administration, which makes surgery difficult. However, to date, there have not been any studies in a large series investigating the changes 
in the thyroid volume of patients with Graves' disease based on a comparison of volumes before and after iodide administration. In this study, therefore, we retrospectively investigated whether and how preoperative excess iodide administration affects thyroid volume in Graves' disease.

\section{Patients and Methods}

\section{Patients}

Between March 2006 and February 2008, 348 patients underwent initial surgical treatment for Graves' disease. In our department, all patients underwent ultrasonography and their thyroid volumes were measured 1-3 days before surgical treatment. Of these 348 patients, 103 who had other thyroid diseases such as benign or malignant nodules and primary hyperparathyroidism, 73 whose thyroid volumes were not measured within 6 months before surgery, and 17 who were administered potassium iodide (KI) for longer than 1 month were excluded from our series. Furthermore, 37 patients whose thyroid volumes exceeded $200 \mathrm{~cm}^{3}$, and 5 who demonstrated a higher TSH level than normal, and were also deleted from the study. This is because it is difficult to accurately evaluate the thyroid volume of patients whose thyroid volume exceeded $200 \mathrm{~cm}^{3}$ on ultrasonography and TSH higher than the normal range could directly affect thyroid enlargement. The remaining 113 patients whose thyroid volumes were previously measured at least one time on ultrasonography within 6 months before surgery were enrolled in this study.

\section{Administration of iodide and evaluation of thyroid volume}

Anti-thyroid drugs (ATD) were administered to all patients. Whether or not KI would also be administrated was individually decided by surgeon. Of these 113 patients, 89 were administered KI ranging from 38 to $114 \mathrm{mg}$ per day for 7 to 18 days before surgery and classified as $\mathrm{KI}(+)$ patients. The average dose of KI per day, total dose of KI, and dosing periods of $\mathrm{KI}(+)$ patients were $64.6+18.3 \mathrm{mg}, 729.7+344 \mathrm{mg}$, and $11.0+3.7$ days, respectively. The applied dose of $\mathrm{KI}$ and dosing periods were decided by surgeons on a case-by-case basis depending on the thyroid function of each patient. There was no iodide administered to the remaining 24 patients, who were classified as $\mathrm{KI}(-)$ patients. The thyroid volume of each subject was measured by high resolutional ultrasonic scanner (Aplio 80, Toshiba Medical Systems, Tokyo, Japan) and calculated as described previously [12].

\section{Statistical analysis}

Statistical analysis was performed using StatView 5.0. The chi-square test, Man-Whitney U test and student $t$ test were adopted for comparing variables. $P$ values less than 0.05 were considered significant.

\section{Results}

We retrospectively investigated changes in thyroid volumes between the two measurement points; the first measurement point, before or during the administration of ATD for KI (-) patients and also before administration of $\mathrm{KI}$ for $\mathrm{KI}(+)$ patients, which was within 6 months before surgery; the second measurement point, after the administration of ATD for KI(-) patients and also after the administration of $\mathrm{KI}$ for $\mathrm{KI}(+)$ patients, which was 1-3 days before surgery. The average period between the first measuring point and surgery were $2.5+1.3$ months for $\mathrm{KI}(+)$ patients and 3.0 +1.3 months for KI(-) patients, respectively, and there was no significant difference between these groups. The thyroid volumes were evaluated on ultrasonography, but ultrasonographic findings did not differ between the two groups. As indicated in Patients and Methods, the applied dose of KI and dosing periods varied among patients, but TSH level, free T4 (FT4) concentration and change in thyroid volume were not related to the total dose of KI (data not shown).

Table 1 summarizes the backgrounds and clinical features of $\mathrm{KI}(+)$ and $\mathrm{KI}(-)$ patients. Age, gender, TSH receptor antibody (TRAb), average thyroid volume, surgical duration and blood loss during surgery did not differ between $\mathrm{KI}(+)$ and $\mathrm{KI}(-)$ patients. Regarding thyroid functions at the first measuring point, FT4 level did not differ between $\mathrm{KI}(+)$ patients and $\mathrm{KI}(-)$ patients but $\mathrm{TSH}$ level in $\mathrm{KI}(+)$ patients was significantly lower $(\mathrm{p}=0.012)$ than that in $\mathrm{KI}(-)$ patients.

Table 2 indicates the change in FT4 concentration, $\mathrm{TSH}$ level, and thyroid volume in $\mathrm{KI}(+)$ and $\mathrm{KI}(-)$ patients between the two measurement points. $\mathrm{In} \mathrm{KI}(+)$ 
Table 1. Backgrounds and clinical features of $\mathrm{KI}(+)$ and $\mathrm{KI}(-)$ patients

\begin{tabular}{lccc}
\hline & $\mathrm{KI}(-)(\mathrm{n}=24)$ & $\mathrm{KI}(+)(\mathrm{n}=89)$ & \\
\hline Age (years) & $33.8 \pm 11.9$ & $32.4 \pm 12.3$ & N.S. \\
Gender (F/M) & $16 / 8$ & $70 / 19$ & N.S. \\
*TRAb (\%) & $73.4 \pm 24.4$ & $81.9 \pm 15.1$ & N.S. \\
*Thyroid volume $\left(\mathrm{cm}^{3}\right)$ & $85.1 \pm 50.5$ & $104.4 \pm 49.9$ & N.S. \\
*TSH level & $0.492 \pm 0.948$ & $0.078 \pm 0.336$ & 0.012 \\
*FT4 level & $1.40 \pm 1.07$ & $1.83 \pm 1.24$ & N.S. \\
Operation time (min) & $109.6 \pm 19.6$ & $111.3 \pm 26.9$ & N.S. \\
Blood loss (g) & $91.9 \pm 71.2$ & $88.8 \pm 68.4$ & N.S. \\
\hline
\end{tabular}

*At the first measurement point

Table 2. Comparison of changes in FT4, TSH and thyroid volume between the two measurement points in $\mathrm{KI}(+)$ and $\mathrm{KI}(-)$ patients

\begin{tabular}{lccr}
\hline KI $(+)$ patients & First measurement point & Second measurement point & \\
\hline FT4 $(\mathrm{ng} / \mathrm{dl})$ & $1.83 \pm 1.24$ & $0.95 \pm 0.58$ & $<0.001$ \\
TSH $(\mathrm{mIU} / \mathrm{ml})$ & $0.078 \pm 0.336$ & $0.469 \pm 1.146$ & $<0.001$ \\
Thyroid volume $\left(\mathrm{cm}^{3}\right)$ & $104.4 \pm 49.9$ & $114.8 \pm 63.3$ & 0.002 \\
\hline & & & \\
\hline KI $(-)$ patients & First measurement point & Second measurement point & \\
\hline FT4 $(\mathrm{ng} / \mathrm{dl})$ & $1.40 \pm 1.07$ & $0.78 \pm 0.28$ & 0.003 \\
TSH $(\mathrm{mIU} / \mathrm{ml})$ & $0.492 \pm 0.948$ & $1.094 \pm 1.599$ & 0.01 \\
Thyroid volume $\left(\mathrm{cm}^{3}\right)$ & $85.1 \pm 50.5$ & $86.6 \pm 50.6$ & N.S. \\
\hline
\end{tabular}

Table 3. Increase in thyroid volume of $\mathrm{KI}(+)$ and $\mathrm{KI}(-)$ patients between two measurement points

\begin{tabular}{|c|c|c|c|c|c|}
\hline $0 \%$ or decrease & $1-9 \%$ & $10-19 \%$ & $20-29 \%$ & $>30 \%$ & Total \\
\hline \multicolumn{6}{|l|}{$\mathrm{KI}(+)$ patients } \\
\hline $34(38 \%)$ & $15(17 \%)$ & $19(21 \%)$ & $6(7 \%)$ & $15(17 \%)$ & 89 \\
\hline \multicolumn{6}{|l|}{$\mathrm{KI}(-)$ patients } \\
\hline $8(33 \%)$ & $10(42 \%)$ & $3(13 \%)$ & $3(13 \%)$ & 0 & 24 \\
\hline
\end{tabular}

patients, FT4 level significantly decreased and TSH level significantly increased after iodide administration $(\mathrm{p}<0.001)$. Furthermore, the average thyroid volume was significantly larger $(p=0.002)$ after iodide administration compared with that before iodide administration. Also in $\mathrm{KI}(-)$ patients who were treated only with ATD, FT4 level decreased $(p=0.003)$ and TSH level increased $(\mathrm{p}=0.01)$ at the second measurement point compared with that at the first measurement point. Average thyroid volume in $\mathrm{KI}(-)$ patients, however, did not differ between these two measurement points, in contrast to $\mathrm{KI}(+)$ patients.

Table 3 demonstrated the increase in thyroid volume of $\mathrm{KI}(+)$ and $\mathrm{KI}(-)$ patients in more detail. The rate of increase in thyroid volume was larger in $\mathrm{KI}(+)$ patients than that in $\mathrm{KI}(-)$ patients $(\mathrm{p}=0.027)$. Of
$89 \mathrm{KI}(+)$ patients, $40(45 \%)$ showed $10 \%$ or more increase and $15(17 \%)$ showed $30 \%$ or more increase in thyroid volume. Six of $24 \mathrm{KI}(-)$ patients $(25 \%)$ also showed $10 \%$ or more increase in thyroid volume, but none of these patients showed $30 \%$ or more increase.

Of $89 \mathrm{KI}(+)$ patients, preoperative TSH remained below measurement sensitivity in 37. Table 4 shows the change in FT4 and TSH levels and thyroid volume of these patients after iodide administration. Although the TSH level remained low, the average thyroid volume significantly increased $(p=0.008)$ after iodide administration.

We investigated morphological differences using tissue specimens of Hematoxylin \& Eosin staining sections. Findings were compared between the two groups based on nodularity, papillary growth, vacuola- 
Table 4. Change in FT4 and thyroid volume before and after iodide administration for 37 patients whose TSH levels remained below the measurement sensitivity

\begin{tabular}{lccc}
\hline & First measurement point & Second measurement point & \\
\hline FT4 $(\mathrm{ng} / \mathrm{dl})$ & $2.37 \pm 1.25$ & $1.20 \pm 0.68$ & $<0.001$ \\
TSH $(\mathrm{mIU} / \mathrm{ml})$ & $<0.003$ & $<0.003$ & \\
Thyroid volume $\left(\mathrm{cm}^{3}\right)$ & $102.3 \pm 52.0$ & $113.5 \pm 58.9$ & 0.008 \\
\hline
\end{tabular}

Table 5. Relationship between morphological variables and KI administration

\begin{tabular}{|c|c|c|c|c|c|}
\hline & & $\mathrm{KI}(+)$ patients $(\%)$ & $\mathrm{KI}(-)$ patients(\%) & Total & \\
\hline \multirow[t]{3}{*}{ Nodularity } & None & $55(62)$ & $16(66)$ & 71 & \multirow{3}{*}{ N.S } \\
\hline & Weak & $31(35)$ & $8(33)$ & 39 & \\
\hline & Strong & $3(3)$ & 0 & 3 & \\
\hline \multirow[t]{3}{*}{ Papillary growth } & None & $6(7)$ & $3(13)$ & 9 & \multirow{3}{*}{ N.S } \\
\hline & Weak & $68(76)$ & $18(75)$ & 86 & \\
\hline & Strong & $15(17)$ & $3(13)$ & 18 & \\
\hline \multirow[t]{3}{*}{ Vacuolation } & None & $9(10)$ & $2(8)$ & 11 & \multirow{3}{*}{ N.S } \\
\hline & Weak & $68(76)$ & $16(67)$ & 84 & \\
\hline & Strong & $12(13)$ & $6(25)$ & 18 & \\
\hline \multirow[t]{3}{*}{ Lymphocyte infiltration } & None & $35(39)$ & $11(46)$ & 46 & \multirow{3}{*}{ N.S. } \\
\hline & Weak & $48(54)$ & $9(38)$ & 57 & \\
\hline & Strong & $6 \quad(7)$ & $4(17)$ & 10 & \\
\hline \multirow[t]{3}{*}{ Fibrosis } & None & $56(63)$ & $15(63)$ & 71 & \multirow{3}{*}{ N.S. } \\
\hline & Weak & $31(35)$ & $8(33)$ & 39 & \\
\hline & Strong & $2(2)$ & 1 (4) & 3 & \\
\hline Total & & 89 & 24 & 113 & \\
\hline
\end{tabular}

tion, lymphocyte infiltration and fibrosis. As shown in Table 5, there were no morphological differences between patients with and without administration of KI.

\section{Discussion}

In this study, we compared increases in thyroid volume between patients with Graves' disease with and without preoperative iodide administration. Although the rate of increase in thyroid volume varied by case in both groups, the average thyroid volumes were significantly increased in $\mathrm{KI}(+)$ patients but not in $\mathrm{KI}(-)$ patients. Furthermore, $17 \%$ of $\mathrm{KI}(+)$ patients, but none of the KI(-) patients, showed a $30 \%$ or greater increase in thyroid volume. Namba et al. showed volume increase in the normal thyroid following iodide administration, which was associated with serum TSH elevation, showing evidence that hypertrophy of the thyroid was induced by TSH stimulation in response to iodide administration [11]. Our series of $\mathrm{KI}(+)$ patients also showed a significant elevation of TSH level $(\mathrm{p}<0.001)$ after iodide administration. Although TSH levels of KI(-) patients were also elevated between the two measurement points, probably due to ATD administration, the difference was less significant than that of KI(-) patients $(p=0.01)$. Since thyroid volume did not change in $\mathrm{KI}(-)$ patients, it is possible that TSH elevation plays a role in the thyroid volume increase with Graves' disease, similar to that in the normal thyroid.

However, we also showed that thyroid volume was significantly increased in $\mathrm{KI}(+)$ patients whose preoperative TSH levels remained below the measurement sensitivity. It is therefore suggested that thyroid volume increase can occur for reasons unrelated to TSH stimulation. Yamada et al. showed that, in human follicular cells, a high concentration of iodide not only de- 
creased the expression of certain angiogenetic factors but also increased the expression of VEGF-C gene, a lymphangiogenesis factor, possibly via the mediation of tumor growth factor (TGF)- $\beta$ [10]. Therefore, it is speculated that hyperplasia of the lymph channel may play a role in the increase of thyroid volume in patients with Graves' disease. However, in our histological study using $\mathrm{H} \& \mathrm{E}$ sections, we could not find any morphological difference between patients with and without KI administration. Therefore, the mechanisms of thyroid enlargement by iodide administration as well as the reasons for individual variations in the change in thyroid volumes of patients belonging to both groups remain unclear and further histological and molecular studies are required to elucidate them.

Although previous reports demonstrated that iodide administration reduces blood flow in the thyroid [3-9], our data showed that surgical duration and blood loss did not differ between $\mathrm{KI}(+)$ and $\mathrm{KI}(-)$ patients, indicating that it is doubtful whether iodide administration always makes surgery easier by reducing intraoperative bleeding. Meanwhile, we demonstrated a significant increase in the thyroid volume of Graves' disease patients elicited by preoperative iodide administration. Therefore, iodide administration may make surgery even more difficult in certain cases because of the thyroid enlargement. Furthermore, since 17\% of $\mathrm{KI}(+)$ patients but none of $\mathrm{KI}(-)$ patients showed a $30 \%$ or greater increase in thyroid volumes, careless iodide administration for a huge goiter may even promote a risk of asphyxia due to the narrowed airway. Therefore, surgeons should consider the application of iodide administration for Graves' disease and be careful when administering iodide for Graves' disease patients especially those with a large goiter.

\section{References}

1. Rienhoff WF (1925) The histological changes brought about in cases of exphthalmic goiter by the administration of iodine. Bull Johns Hopkins Hosp 37: 285-306.

2. Plummer HS (1923) Results of administering iodine to patients having exophthalmic goiter. JAMA 80: 1955.

3. Arntzenius AB, Smig LJ, Schipper J, van der Beide D, Meinders AE (1991) Inverse relation between iodine intake and thyroid blood flow: color Doppler flow imaging in euthyroid humans. J Clin Endocrinol Metab 73: 1051-1055.

4. Marigold JH, Morgan AK, Earle DJ, Young AE, Croft DN (1985) Lugol's iodine: Its effect on thyroid blood flow in patients with thyrotoxicosis. Br J Surg 72: 45-47.

5. Chang DC, Wheeler MH, Wood cock JP, Curley I, Lazarus JR, Fung H, John R, Hall R, McGregor AM (1987) The effect of preoperative Lugol's iodine on thyroid blod flow in patients with Graves' hyperthyroidism. Surgery 102: 1055-1061.

6. Kaur S, Parr JH, Ramsay ID, Hennebry TM, Jarvis KJ, Lester E (1988) Effect of preoperative iodine in patients with Graves' diseases controlled with antithyroid drugs and thyroxine. Ann R Coll Surg Engl 70: 123127.

7. Rodier JF, Janser JC, Petit H, Schneegans O, Ot G, Kaissling A, Grob JC, Velten M (1998) Effect of preoperative administration of Lugol solution on thyroid blood flow in hyperthyroidism. Ann Chir 52: 229-233.
8. Ansaldo GL, Pretolesi F, Varaldo E, Meola C, Minuto M, BOrgonovo G, Derchi LE, Torre QC (2000) Doppler evaluation of intrathyroid arterial resistances during preoperative treatment with Lugol iodide solution in patients with diffuse toxic goiter. $\mathrm{J} \mathrm{Am} \mathrm{Coll}$ Surg 191: 607-612.

9. Erbil Y, Ozluk Y, Giris M, Salmashoglu A, Issever H, Barbaros U, Kapran Y, Ozarmagan S, Tezelman S (2007) Effect of Lugol solution on thyroid gland blood flow and microvessel density in the patients with Graves' disease. J Clin Endocrinol Metab 92: 21822189.

10. Yamada E, Yamazaki K, Takano K, Obara T, Sato K (2006) Iodide inhibits vascular endothelial growth factor - A expression in cultured human thyroid follicules: a microarray search for effects of thyrotropin and iodide on angiogenesis factors. Thyroid 16: 545-555.

11. Namba H, Yamashita S, Kimura H, Yokoyama N, Usa T, Otsuru A, Izumi M, Nagataki S (1993) Evidence of thyroid volume increase in normal subjects receiving excess iodide. J Clin Endocrinol Metab 76: 605-608.

12. Murakami Y, Takamatsu J, Sakane S, Kuma K, Ohsawa $\mathrm{N}$ (1996) Changes in thyroid volume in response to radioactive iodine for Graves' hyperthyroidism correlated with activity of thyroid-stimulating antibody and treatment outcome. J Clin Endocrinol Metab 81: 3257-326. 